\title{
Laboratory and pilot plant scale study on water dechlorination by medium pressure ultraviolet (UV) radiation
}

\author{
Maryani Paramita Astuti ${ }^{1}$, Rongjing $\mathrm{Xie}^{2}$, and Nicky Satyadharma Aziz ${ }^{3}$ \\ ${ }^{1}$ Environmental Engineering Study Program Faculty of Engineering, President University, 17550 Cikarang Indonesia \\ ${ }^{2}$ Water Supply (Plants) Department, PUB, 228231 Scotts Road, Singapore \\ ${ }^{3}$ Infrastructure Civil Department, Surbana Jurong Consultants Pte. Ltd, 150168 Bukit Merah, Singapore
}

\begin{abstract}
Ultra violet (UV) dechlorination was performed to eliminate residual chlorine as the byproducts of chlorination process. UV dechlorination utilizes photon energy generated by medium pressure (MP) UV lamp to produce powerful hydroxyls which in return break down chemical bond of the residual chlorine. This study was undertaken to investigate the removal of residual chlorine under a medium pressure UV radiation and evaluate the influence of UV fluence on chlorine removal efficiency based on both laboratory and plant scale experiments. In laboratory experiments, water samples were exposed to a UV collimated beam apparatus equipped with a medium pressure (MP) UV lamp over a specified exposure time. Chlorine concentrations were measured before and after UV exposure to calculate its chlorine removal efficiency. Results showed that chlorine residual decreased over time and the removal efficiency increased as the UV fluence increased. The maximum UV fluence applied in the laboratory experiments $(513 \mathrm{~mJ} / \mathrm{cm} 2)$ resulted in less than $25 \%$ of total chlorine reduction. The field experiments were conducted at a waterworks in Singapore with total capacity of $22 \mathrm{l} / \mathrm{s}$ using an existing UV system. The plant scale studies supported laboratory findings with about $9 \%$ of chlorine removal efficiency. The chlorine decay rates (fluence-based first order constant) were also calculated.
\end{abstract}

\section{Introduction}

Chlorination remains as the most widely used method for disinfection of water and wastewater in the United State [1] and probably worldwide. Some advantages of this process include: (1) relatively low cost, (2) high efficiency, and (3) ease of use [2]. In this process, chlorine is commonly introduced in the forms of chlorine gas $\left(\mathrm{Cl}_{2}\right)$, sodium hypochlorite $(\mathrm{NaOCl})$, or calcium hypochlorite $\left(\mathrm{Ca}(\mathrm{OCl})_{2}\right)$. When each of these chemical is added to water, rapid hydrolysis occurs to form hypochlorous acid, $\mathrm{HOCl}$, which is further hydrolyzed to yield hypochlorite ion, $\mathrm{OCl}^{-}$, as shown in the reaction (1) through (4). Reaction (4) is strongly_dependent on $\mathrm{pH}$ of the solution with equilibrium constant $\mathrm{pKa}=7.5$ (at $25^{\circ} \mathrm{C}$ ) [3]. Both hypochlorous acid and hypochlorite ions are commonly referred to as "free chlorine residual" [2].

$$
\begin{aligned}
\mathrm{Cl}_{2}+\mathrm{H}_{2} \mathrm{O} & \rightarrow \mathrm{HOCl}+\mathrm{H}^{+}+\mathrm{Cl}^{-} \\
\mathrm{NaOCl}+\mathrm{H}_{2} \mathrm{O} & \rightarrow \mathrm{Na}^{+}+\mathrm{HOCl}+\mathrm{OH} \\
\mathrm{Ca}(\mathrm{OCl})_{2}+2 \mathrm{H}_{2} \mathrm{O} & \rightarrow \mathrm{Ca}^{+}+2 \mathrm{HOCl}+2 \mathrm{OH}^{-} \\
\mathrm{HOCl} & \leftrightarrow \mathrm{H}++\mathrm{OCl}^{-}
\end{aligned}
$$

In the presence of ammonia, hypochlorous acid and hypochlorite ion react with the ammonia to form monochloramine $\left(\mathrm{NH}_{2} \mathrm{Cl}\right)$, dichloramine $\left(\mathrm{NHCl}_{2}\right)$, and trichloramine $\left(\mathrm{NCl}_{3}\right)$, which referred to as "combined chlorine residual". Chlorination that converts all ammonia to either trichloramine or nitrogen gas is known as "break point chlorination". After the break point, all ammonia is converted and thus the addition of chlorine resulted in formation of free chlorine residual.

Despite the extensive use of chlorination, chlorine residual is of concern to drinking water and wastewater treatment. Chlorine generates unpleasant odor and affects taste of drinks and liquids [4]. Recent studies suggest that residual chlorines may react further with dissolved organic matter by oxidation, addition and substitution reactions to form a range of DBPs (disinfection by products) such as the trihalomethanes (THMs), haloacetic acids (HAAs), and chlorite [5] that may pose unintended health hazards. Whilst for industrial water, chlorine residual could damage delicate process equipment such as reverse osmosis (RO) and deionization (DI) resin units and could accelerate corrosion of vessels, valves and piping.

In the case of wastewater treatment, toxicity of chlorine to aquatic life is another concern which had been studied extensively during the late 1970s and early 1980s. Further research on chlorine toxicity on early life 
stages of freshwater mussels showed that the impact of long-term exposure to low doses of chlorine may result in sub-lethal impairment [6]. Review of literature [7] concludes that DBPs formed in chlorinated wastewaters can be toxic and may have a deleterious impact on aquatic organisms. Therefore, water dechlorination process is needed to remove residual chlorine to meet specified standard of the treated water.

Ultraviolet (UV) dechlorination utilizes photon energy generated by low pressure (LP) and medium pressure (MP) UV lamps that contain an inert gas, e.g., argon, and a small amount of liquid mercury [8]. When a potential energy is applied to the lamp, some of the liquid mercury vaporizes and collides with free electrons and ions, and thus increases the energy state of mercury vapor which tends to return to its normal energy state by discharging energy in the form of UV light [9]. This discharged energy is then used to break down chemical bond of the residual chlorine. The LP lamps emit essentially monochromatic light at $254 \mathrm{~nm}$ wavelength, whilst the MP lamps radiate polychromatic lights ranging from about $205 \mathrm{~nm}$ to above $500 \mathrm{~nm}$ wavelength [8].

In the photodecomposition reactions of free chlorine in water, various primary reactive intermediates are formed which are rapidly converted into hydroxyl radical $(\bullet \mathrm{OH})$ and chlorine radical $(\cdot \mathrm{Cl})$. These reactions are presented in reaction (5) through reaction (8). The rate of free chlorine destruction under UV irradiation is best estimated by a first-order kinetics [10].

$$
\begin{gathered}
\mathrm{HOCl}+\text { uv photons } \rightarrow \cdot \mathrm{OH}+\cdot \mathrm{Cl} \\
\mathrm{OCl}^{-}+\text {uv photons } \rightarrow \cdot \mathrm{OH}+\cdot \mathrm{Cl} \\
\cdot \mathrm{O}^{-}+\mathrm{H}_{2} \mathrm{O} \rightarrow \cdot \mathrm{OH}+\mathrm{OH}^{-}
\end{gathered}
$$

UV dechlorination is believed to outperform other dechlorination technologies since there is no mixing or contact tanks required, no chemical addition required, and only minimum operation and maintenance involved. It leaves no impact on the taste, odor, color, and $\mathrm{pH}$ of water as well. However, chlorine removal efficiency needs to be considered prior to applying this method. Therefore, this study was undertaken to investigate the effectiveness of a medium pressure UV technology in water dechlorination and evaluate the influence of UV fluence dose on chlorine removal efficiency based on laboratory experiments and pilot plant scale experiments. In addition, based on the experimental results obtained in this study, fluence-based chlorine decay rates under UV exposure were determined.

\section{Experimental details}

Water samples containing an equal total chlorine concentration were exposed to polychromatic UV irradiation produced by medium pressure (MP) UV lamps. MP lamps were operated at temperatures range of $600-900^{\circ} \mathrm{C}$ and at mercury vapour pressures of $2-200$ psi [9]. At the beginning and end of the UV exposure, the total and free chlorine concentration in each sample was measured in order to calculate the amount of chlorine removed due to UV exposure.

The experiments were carried out in laboratory scale and in pilot plant scale. Water samples used in the experiments were the drinking water produced by a waterworks in Singapore. All experiments were conducted at ambient temperature $\left(23 \pm 2^{\circ} \mathrm{C}\right)$.

The initial total chlorine concentration was designed at $2 \mathrm{mg} / \mathrm{L}$. Since chlorine is a highly oxidative compound, an equal chlorine concentration was difficult to achieve. Therefore, the concentrations were set of to be within the range of $1.90 \mathrm{mg} / \mathrm{L}$ to $2.2 \mathrm{mg} / \mathrm{L}$.

\subsection{Laboratory scale experiments}

The laboratory experiments were conducted at CAWT (Centre for Advanced Water Technology) Laboratory in Singapore using a collimated beam UV apparatus (Atlantium Technologies, Ltd) equipped with an MP UV lamp. Each sample was placed in a 6 - $\mathrm{cm}$ petri dish and was exposed to UV light over specified exposure time. A controlled experiment was designed, i.e., a sample which was placed in the dark on a bench far-off from the UV apparatus over the duration of the experiment.

Schematic of the laboratory experiments set up is shown in Figure 1. The surface of the samples to be exposed was about $2 \mathrm{~cm}$ from the UV apparatus. Appropriate amounts from a sodium hypochlorite dosing solution made from a sodium hypochlorite solution $(\mathrm{NaOCl} 12 \%)$ were added to the water samples to obtain the desired initial chlorine concentration. The chlorine concentrations were measured before and after UV exposure by a DPD colorimeter (HACH DR/890).

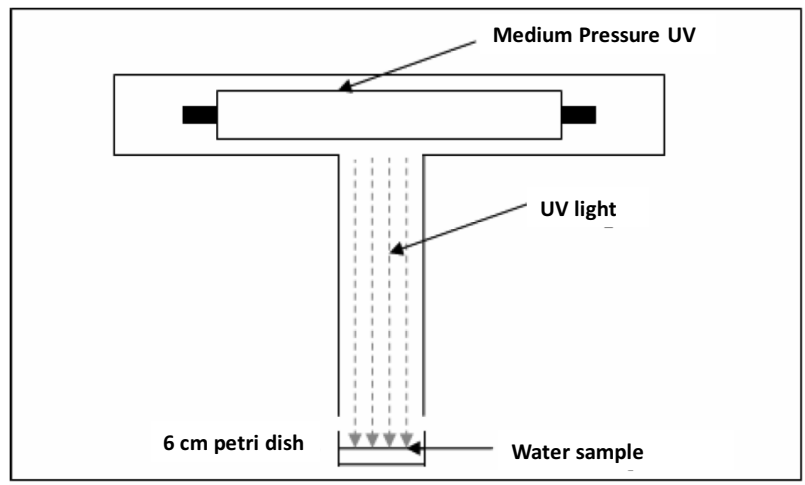

Fig. 1. Schematic of laboratory experiments

\subsection{Pilot plant scale experiments}

The pilot plant scale (with a capacity of $22 \mathrm{~L} / \mathrm{s}$ ) experiments were performed at a waterworks in Singapore. The raw water of this waterworks was extracted from reservoirs and then treated which would then be treated via coagulation, sedimentation and sand filtration, followed by chlorination as disinfection unit.

The UV system used in the experiments was Atlantium Hydro-Optic R-200. The sodium hypochlorite dosing solution was made from a commercial solution of 
sodium hypochlorite $(\mathrm{NaOCl} 12 \%)$. This dosing solution was injected at the inlet of the UV system. The chlorine concentrations were then measured at the inlet after the inline static mixer and at the outlet of the UV system by a DPD colorimeter (HACH DR/890).

\subsection{Chlorine residual analysis}

In the experiments, concentration of total and free chlorine were measured by N,N-diethyl-pphenylenediamine (DPD) colorimetric method using Hach pocket chlorine colorimeters (DR/890) with two types of reagents-DPD Free Chlorine Reagent and DPD Total Chlorine Reagent-for 10-ml of sample reagent.

The DPD amine (reagent) was added to the water sample and was oxidized by chlorine contained in the sample to two oxidation products, Würster dye and imine. Würster dye was relatively stable and would form a magenta color at neutral $\mathrm{pH}$, whilst imine was relatively unstable and colorless that would be formed at higher oxidant levels, i.e., higher chlorine concentration. The intensity of the magenta color was then measured photo-metrically which represented concentration of chlorine in the samples. The DPD-chlorine reactions are illustrated in Figure 2.

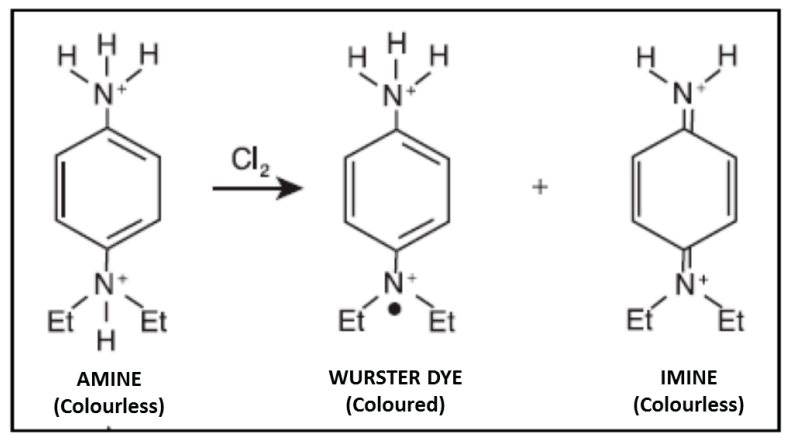

Fig. 2. DPD-chlorine reaction [12]

\subsection{UV fluence variations}

UV fluence refers to multiplication of UV intensity by the exposure time. The UV intensity of the UV collimated beam used in the laboratory experiments was set at a fixed value, UV fluence variations were achieved by varying exposure times of the samples to UV beams. The time variations were 10,20 , and 30 minutes. The fluence was measured by a radiometer Model SEL240 UV Curing Radiometer, Internal Light Inc. This device consists of a highly sensitive electronic ammeter connected to a UV sensor head, which generates a current proportional to the incident fluence rate. The device was used to measure UV intensity of a collimated beam apparatus by setting its radiometer detector head at the same height as the surface of samples in petri dish. Results of the UV intensity of the UV beam are presented in Figure 3.

In the experiments, $2 \mathrm{~cm}$ distance of the sample surface and the UV apparatus was selected, which per Figure 3 was equal to the UV intensity of 0.285
$\mathrm{mW} / \mathrm{cm}^{2}$. The UV fluence was calculated by multiplying UV intensity by the exposure time, which were 171, 342, and $513 \mathrm{~mJ} / \mathrm{cm}^{2}$.

In the pilot plant scale experiments, UV fluence were 80,130 , and $180 \mathrm{~mJ} / \mathrm{cm}^{2}$. For each of the trials, the UV system-Atlantium Hydro-Optic R200-was adjusted automatically to the specified UV fluence by adjusting the UV intensity value.

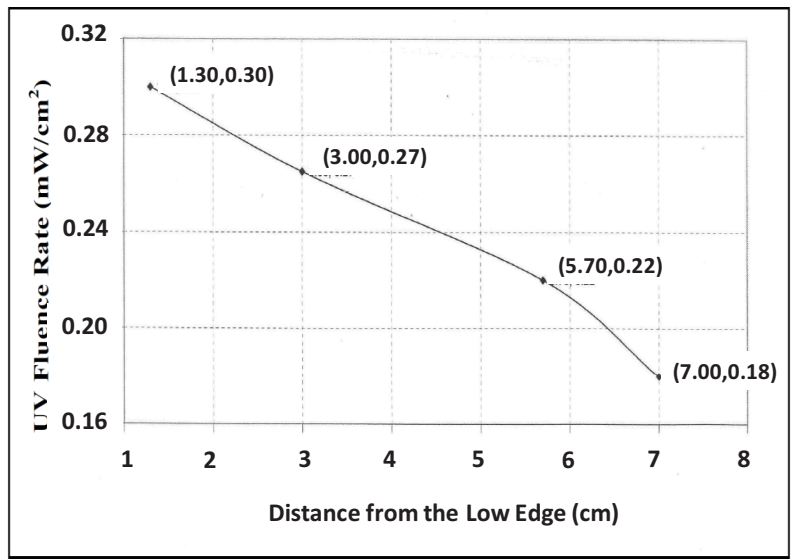

Fig. 3. UV intensity of collimated beam apparatus at varied exposure distance

\subsection{Chlorine decay rates (fluence-based first order rate constant) analysis}

Results from the experiments were used to calculate chlorine decay rates assuming a first order kinetics. Reference [10] states the rate of chlorine destruction under UV exposure was best estimated by a first-order kinetics which is usually expressed as:

$$
\ln \frac{[C]_{o}}{[C]_{t}}=k_{1}{ }^{\prime} t
$$

Where $[C]_{o}$ and $[C]_{t}$ are the chlorine concentration before and after exposure to UV, respectively for a time of $\mathrm{t}$ min; $\mathrm{k}$ is the first order constant in unit of $\mathrm{min}^{-1}$.

Since the value of $\mathrm{k}$ is affected by the experimental conditions (e.g., irradiance, absorbance, path length, and etc) which are difficult to reproduce, this expression can be modified into:

$$
\begin{aligned}
& \ln \frac{[C]_{o}}{[C]_{F}}=k_{1}^{\prime} F \\
& \frac{[C]_{F}}{[C]_{o}}=e^{-k_{1}^{\prime} F}
\end{aligned}
$$

where $[C]_{o}$ and $[C]_{F}$ are the chlorine concentration before and after exposure to the UV fluence of $F ; k_{1}^{\prime}$ is the fluence-based first order rate constant [10].

\section{Results and discussions}

\subsection{Chlorine removal efficiencies}

Results obtained from both laboratory and plant scale experiments indicate that chlorine concentration 
decreases upon exposure of UV light. The total chlorine removal efficiencies illustrated in Figure 4 suggest that the removal efficiency increases as UV fluence increases. It is because higher fluence transfers greater amounts of energy to the samples which then utilized to break the chemical bonds of residual chlorine in the samples. These results are consistent with those reported $[10,13,14]$, suggesting that higher UV fluence is required to achieve higher removal efficiencies.

The control experiments (dark sample) showed small decreases in chlorine concentration $(<4.8 \%)$. These amounts of chlorine are considered as chlorine demand of the water sample, i.e., the amount of chlorine required to remove impurities in the water sample. This suggests that the removal efficiencies achieved in this study are inclusive of chlorine demand of the water samples.

As shown in Figure 4, the highest UV fluence utilized in the laboratory experiments, $513 \mathrm{~mJ} / \mathrm{cm}^{2}$, results in about $25 \%$ of total chlorine removal efficiency. The pilot plant scale experiments with typical UV fluence dose resulted in only about $9 \%$ of maximum removal efficiency at the highest UV fluence of 180 $\mathrm{mJ} / \mathrm{cm}^{2}$. The graph also illustrates that the removal efficiencies yield in laboratory experiments were generally higher than those achieved in pilot plant experiments.

At about the same UV fluence, i.e., $171 \mathrm{~mJ} / \mathrm{cm}^{2}$ in laboratory scale and $180 \mathrm{~mJ} / \mathrm{cm} 2$ in pilot plant scale, the removal efficiencies achieved in laboratory scale are about $17 \%$ whilst the pilot plant scale only resulted in about $10 \%$. This likely occurred because the water sample was in contact with the open air in the laboratory scale, whereas in pilot plant scale set up the water sample was inside the inlet pipe and UV reactor at all times. Therefore, some loss of chlorine in the laboratory scale experiment could be attributed to the relative volatility and instability of chlorine compounds in water. Further studies are needed to confirm this hypothesis.

Watts and Linden [14] showed that nitrate, $\mathrm{NO}_{3}$, is among the products formed by UV reactions of water containing monochloramine and under some conditions, photolysis of nitrate led to formation of nitrite, $\mathrm{NO}_{2}$. Such products are considered as drinking water, surface water, and groundwater contaminants, which pose threat to human health upon consumption. In fact, The National Drinking Water Quality Standard released by USEPA sets the MCL (maximum contaminant level) of nitrate at $10 \mathrm{mg} / \mathrm{L}$ and nitrite at $1 \mathrm{mg} / \mathrm{L}$.

The formation of these compounds in deionized (DI) water during photolysis of aqueous monochloramine as shown in Figure 5 indicates that concentration of nitrite and nitrate formed by the photolysis reaction increases as the UV fluence increases.

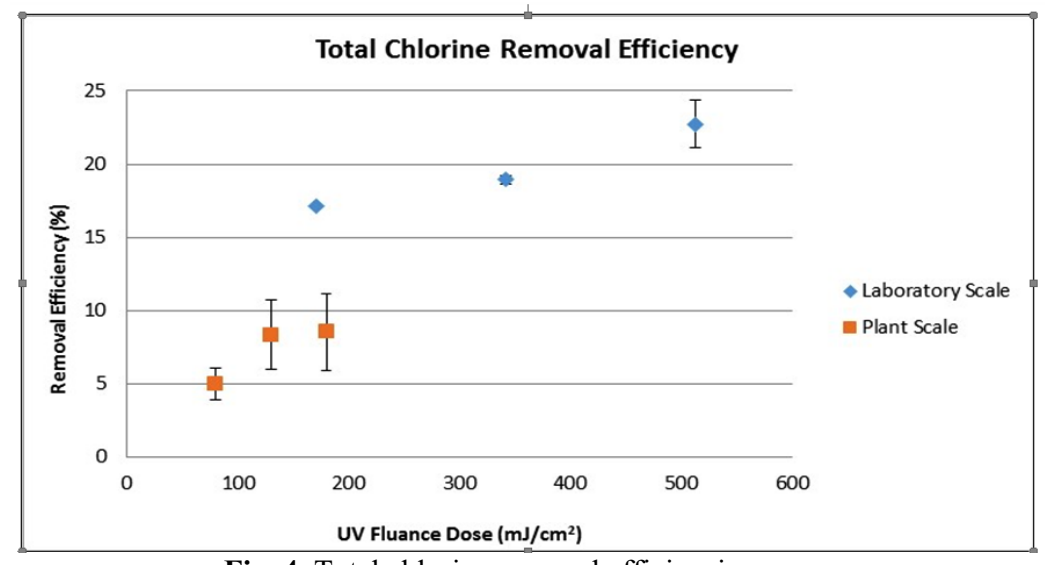

Fig. 4. Total chlorine removal efficiencies

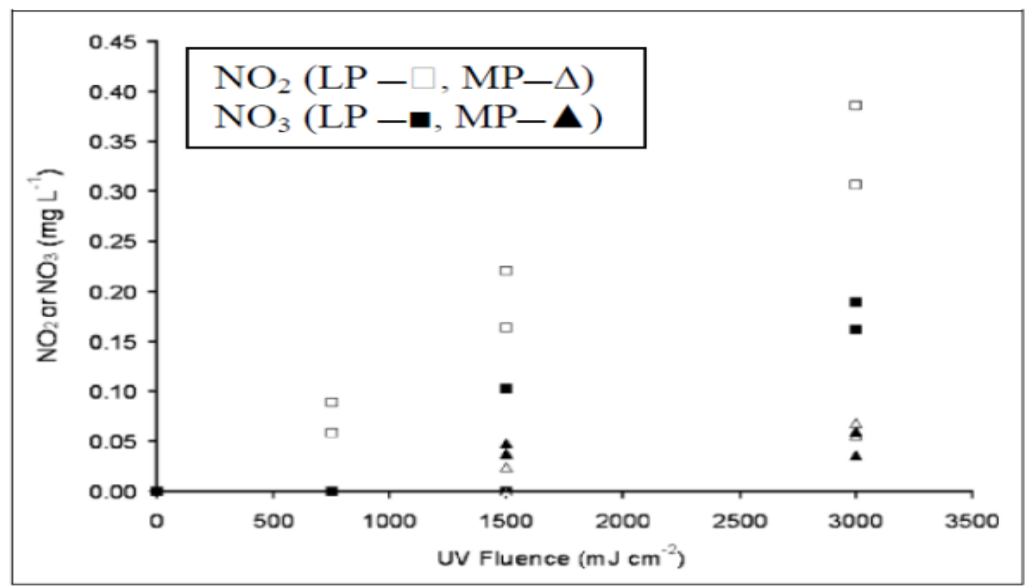

Fig. 5. Nitrogenous photoproduct formation in chloraminated $(4.5 \mathrm{mgCl} / \mathrm{L})$ DI water after $\mathrm{UV}$ exposure [14] 


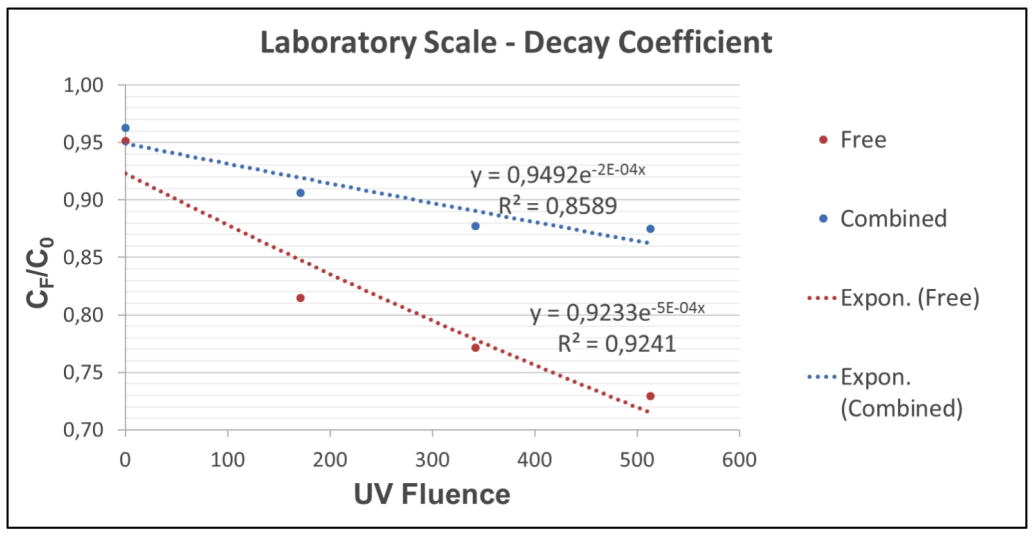

Fig.6. Chlorine decay constant - laboratory scale

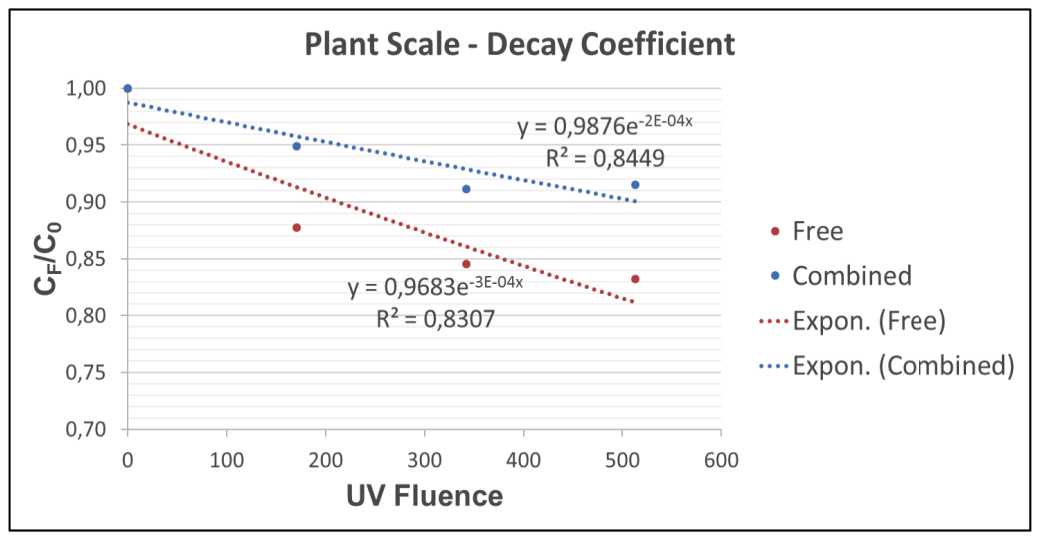

Fig.7. Chlorine decay constant - pilot plant scale

Therefore, further study conducted to investigate the required UV fluence to achieve better removal efficiencies should consider the formation of these compounds. Figure 5 also shows that there was little nitrate and nitrite formation in the range of UV fluence applied in this study.

\subsection{Chlorine Decay Rates (fluence-based first order rate constant)}

Results of chlorine decay constant from laboratory scale (Figure 6) and pilot plant scale (Figure 7) showed that the decay rates of free chlorines were higher than those of combined chlorines. This finding suggests that polychromatic UV light used in the experiments is more effective in decaying free chlorine than combined chlorine.

This is likely due to the maximum photon absorption of free chlorine at $290 \mathrm{~nm}$. Such wavelength falls in the range of polychromatic UV light spectrum by the MP UV lamp. However, monochloramine's maximum photon absorbance is approximately at 246 $\mathrm{nm}$ which is close to the wavelength of monochromatic spectrum produced by LP UV Lamp [13]. The $\mathrm{R}^{2}$ values of correlations from the experiments data are greater than 0.83, revealing near significant relationship although more data points were preferred to obtain better $\mathrm{R}^{2}$ values and to accurately determine the kinetic reactions of free and combined chlorine under UV light exposure.

\section{Conclusions}

In this study, the effectiveness of a medium pressure UV technology in water dechlorination was investigated in laboratory scale and plant scale experiments. The results proved that UV light radiation reduces chlorine concentration in water samples. Higher UV fluence dose generated higher total chlorine removal efficiencies. The highest UV fluence dose applied in the laboratory scale experiments, i.e., 513 $\mathrm{mJ} / \mathrm{cm}^{2}$, resulted in about $25 \%$ of chlorine removal, whilst the maximum fluence dose utilized in the plant scale experiments, i.e., $30 \mathrm{~mJ} / \mathrm{cm}^{2}$, resulted in about $9 \%$ of removal efficiency. The fluence-based free and combined chlorine decay rates were found and the results show that under exposure of polychromatic UV light generated by MP UV lamp, free chlorine was degraded faster than combined chlorine.

The authors would like to thank Nanyang Technological University (NTU) and Center for Advanced Water Technology (CAWT) for research facilities used in this project. Dr. Benoit Jean Guieysse, Professor at Massey 
University of New Zealand (previously Assistant Professor at NTU) for his valuable guidance and support, Mylene Gomez, $\mathrm{Fu}$ Yong, and other researchers researchers at CAWT laboratory for their timely assistance and technical support.

\section{References}

1. J.K. Howe, D.W. Hand, J.C. Crittenden, R.R. Trussell, G. Tchobanoglous,: John Wiley \& Sons, (2012)

2. D.C. Gang, T.E. Clevenger, S.K. Banerji, J. Env. Informatics, 1, 21 (2003)

3. G. Tchobanoglous, F.L. Burton, H.D. Stensel, Metcalf and Eddy. McGraw-Hill Education, (2003)

4. Spellman, R. Frank, Handbook of Water and Wastewater Treatment Operations, Boca Raton: CRC Press, (2013)

A. Herbert, D. Forestier, D. Lenes, D. Benanou, S. Jacob, C. Arfi, L. Lambolez, Y. Levi, J. Water Rsch, 44, 3147 (2010)
5. T.W. Valenti, D.S. Cherry, R.J. Currie, R.J. Neves, J.W. Jones, , J. Env. Toxicology and Chemistry. 25, 2512 (2006)

6. K. Watson, G. Shaw, F.D.L. Leusch, N.L. Knight, J. Water Rsch. 46, 6069 (2012)

7. K.G. Linden, C.M. Sharpless, J. Env. Sci. Tech, 37, 1933 (2003)

8. S.H. Clarke, U.S. Army Center for Health Promotion and Preventive Medicine, (2006)

9. Y. Feng, D.W. Smith, J.R. Bolton, J. Env. Eng. Sci, 6, 277 (2007)

10. J.R. Bolton, Ultraviolet Applications Handbook, Edmonton: ICC Lifelong Learn Inc, (2010)

11. D.L. Harp, Technical Information Series--Booklet No17, (2002)

B. Örmeci, G.A. Ishida, K.G. Linden, J. Water SRT Aqua, 54 (2005)

12. M.J. Watts, K.G. Linden, J. Water Rsch, 41, 2871 (2007)

13. R.J. Xie, M.J. Gomez, Y.J. Xing, J. Env. Sci. Hlth A Toxicity Hazard Substances, 42, 2047(2007) 